\title{
Heat and mass transfer at sublimation of a single chromium (III) and zirconium (IV) $\beta$-diketonate particle in the inert gas mixture
}

\author{
Igor Igumenov ${ }^{1}$, Maksim Makarov ${ }^{2}$, and Sofya Makarova ${ }^{1,2, *}$ \\ ${ }^{1}$ Nikolaev Institute of Inorganic Chemistry, SB RAS, 3 Lavrentiev ave., Novosibirsk 630090, Russia \\ ${ }^{2}$ Kutateladze Institute of Thermophysics, SB RAS, 1 Lavrentiev ave., Novosibirsk 630090, Russia
}

\begin{abstract}
The paper proposes a physical and mathematical model of nonstationary sublimation of single spherical particles of volatile chromium (III) and zirconium (IV) $\beta$-diketonates, floating in the flow of a binary helium-argon mixture. The influence of the carrier gas composition on the kinetics of sublimation has been analyzed. The addition of helium to the carrier gas is shown to increase the intensity of sublimation.
\end{abstract}

\section{Introduction}

Heat and mass transfer at sublimation of solid particles is important for optimizing the operation modes of cryogenic systems, for intensifying the low-temperature drying of pharmaceuticals and food products, as well as in the development of new technologies of chemical vapor deposition (CVD). The technology of applying ceramic thermal barrier coatings is one of the key challenges when creating gas turbine engines. A method of chemical deposition from the gas phase using volatile compounds of metals with organic ligands (MO CVD) is promising for obtaining such coatings [1,2]. At that, the technology has to ensure a sufficiently high growth rate of the coating while maintaining the columnar structure. The necessary high concentration of precursor in the vapor phase can be achieved through sublimation under the conditions of intensive heat and mass transfer. High intensity of heat supply to the surface of particles suspended in an inert carrier gas may be obtained in a centrifugal fluidized bed of large inert particles in the vortex chamber [3]. For the optimum design of the vortex chamber the essential is the kinetics of substance sublimation, depending on the thermodynamic and transport properties of the precursor and carrier gas.

This paper presents the physical and mathematical model of sublimation of a single particle of the metalorganic compound, floating in a binary mixture of inert gases. The influence of the carrier gas composition on the dynamics of the sublimation process has been analyzed.

\footnotetext{
* Corresponding author: snmakarova@itp.nsc.ru
} 


\section{Problem statement}

The considered phenomenon is nonstationary sublimation of a single spherical particle of the precursor (chromium (III) acetylacetonate $\mathrm{Cr}(\mathrm{aa})_{3}$ or zirconium (IV) dipivaloyl-methane $\left.\mathrm{Zr}(\mathrm{dpm})_{4}\right)$. The sublimation is believed to occur uniformly over the entire surface of the solid particle, and the bulk sublimation inside the pores of the material is not taken into account. The precursor particle does not lose its spherical symmetry over time.

At low velocities of streamlining (diffusion mode of sublimation) the processes of heat and mass transfer in the gas phase are described by a system of parabolic partial differential equations of heat conduction and diffusion of the second order:

$$
c_{p}^{g} \frac{\partial\left(\rho^{g} T\right)}{\partial \tau}=\frac{1}{r^{2}} \frac{\partial}{\partial r}\left(r^{2} \lambda^{g} \frac{\partial T}{\partial r}\right)+\sum_{i=1}^{n}\left(-J_{i r} c_{p i}^{g}\right) \frac{\partial T}{\partial r} \text { and } \rho^{g} \frac{\partial K_{i}^{g}}{\partial \tau}=\frac{1}{r^{2}} \frac{\partial\left(-r^{2} J_{i r}\right)}{\partial r} .
$$

For multicomponent gas mixtures (three or more components) the diffusion flux of the i-th gas in the mixture depends not only on its own concentration gradient, but also on concentration gradients of remaining components of the mixture. A number of approaches were developed to describe this effect $[4,5]$. The most accurate results are ensured by the approaches based on Maxwell-Stefan relations, namely, models of Krishna, Lapin-Strelets, etc. However, such approaches require significant computational resources. Simplified models based on the concept of effective diffusion coefficient are more economically efficient, but often have a limited scope of application. One of the most effective models of multicomponent diffusion is Ramshaw model [6], which is used in this work. The diffusion flux of each component of the mixture is determined by correlations below:

$$
J_{i r}=-\rho^{g} D_{i} \frac{\partial K_{i}^{g}}{\partial r}+\delta_{J i r} ; \quad \delta_{J i r}=\rho^{g} \frac{D_{i} K_{i}^{g}}{M} \frac{\partial M}{\partial r}-\rho^{g} K_{i}^{g} \sum_{k=1}^{n}\left(-D_{k} \frac{\partial K_{k}^{g}}{\partial r}+\frac{D_{k} K_{k}^{g}}{M} \frac{\partial M}{\partial r}\right) .
$$

The effective diffusion coefficient is calculated according to the formula:

$$
D_{i}=\sum_{\substack{k=1 \\ k \neq i}}^{n} \frac{K_{k}^{g}}{M_{k}} / \sum_{\substack{k=1 \\ k \neq i}}^{n} \frac{K_{k}^{g}}{M_{k} D_{i k}} .
$$

At high velocities of steramlining (convective mode of sublimation) the heat and mass transfer intensity on the particle surface is determined by the Ranz-Marshall similarity equations [7]:

$$
\mathrm{Nu}=2+0.6 \operatorname{Re}_{d}^{0.5} \operatorname{Pr}^{1 / 3}, \mathrm{Sh}=2+0.6 \mathrm{Re}_{d}^{0.5} \mathrm{Sc}^{1 / 3} .
$$

The temperature of the solid particle regardless of the sublimation mode is described by the heat conduction equation in the form:

$$
c_{p}^{s} \rho^{s} \frac{\partial T}{\partial \tau}=\frac{1}{r^{2}} \frac{\partial}{\partial r}\left(r^{2} \lambda^{s} \frac{\partial T}{\partial r}\right) .
$$

The main difficulty in the transition from diffusive to convective approximation is that the gas mixture composition on the particle surface, necessary for calculating the similarity numbers in (1) cannot be determined from the boundary conditions of the problem. For a ternary mixture, a solution to this problem is reduced to defining a separation factor of inert gases. In this work, the separation factor is determined by the results of the precursor sublimation modeling in the diffusion approximation. The obtained data are approximated by a rational polynomial of the second degree, depending on the mass fraction of the precursor vapor on the particle surface, and then are used to calculate the equations (1).

Initial and boundary conditions, determining the size of calculation domain, and testing the solution method for the binary system $\mathrm{Cr}(\mathrm{aa})_{3} / \mathrm{Ar}$ are described in detail in [8]. The mass fraction of vapor of the precursor $K_{1 R_{w}^{+}}^{g}$ at the phase boundary is determined by the temperature of the particle surface and the curve of vapor saturation (sublimation curve). 
According to [9] for chromium (III) acetylacetonate, the sublimation curve is described by the formula: $\ln p=39.197-15308.5 / T$; for zirconium (IV) dipivaloylmethane the data of work [10], supplemented by results of specially conducted research in the IIC SB RAS for a wider range of temperatures, are approximated by the formula: $\ln p=26.652-11006.4 / T$. The pressures and temperatures in these correlations are determined in $\mathrm{Pa}$ and $\mathrm{K}$, correspondently.

\section{Method of solution}

The main feature of the solution method is integration of the written differential equations system in moving coordinates $(\tau, \eta)$, where $\tau$ is the time, $\eta$ is the relative radial coordinate for the area of gas-vapor mixture $\eta^{g}=\left(r-R_{w}\right) /\left(R_{e}-R_{w}\right)$ and the area of a solid particle $\eta^{s}=r / R_{w}$. The position of the phase change boundary $R_{w}$ is determined by the sublimation intensity and is time-dependent. Explicit boundary detection allows minimizing the time of computation and improving the accuracy of the results.

\section{Results of modeling}

Fig. 1 presents data for the separation factor of argon-helium mixture, depending on the precursor vapors fraction at the phase change boundary, obtained in the diffusion approximation. It is shown that there are sublimation modes with a constant ratio of inert components in the gas phase. In the case of sublimation of $\mathrm{Cr}(\mathrm{aa})_{3}$ the consistency of ratios of mass fractions of $\mathrm{Ar}$ and $\mathrm{He}$ is observed at $K_{e}^{A r}=0.9$, for $\mathrm{Zr}(\mathrm{dpm})_{4}-$ at $K_{e}^{A r}=0.8$. At a high concentration of argon in the environment $\left(K_{e}^{A r}=0.95\right)$ the vapor-gas mixture at the phase change boundary is more enriched in helium, and at low concentration it is the other way around. The factor of the mixture separation does not depend on the environment gas temperature.
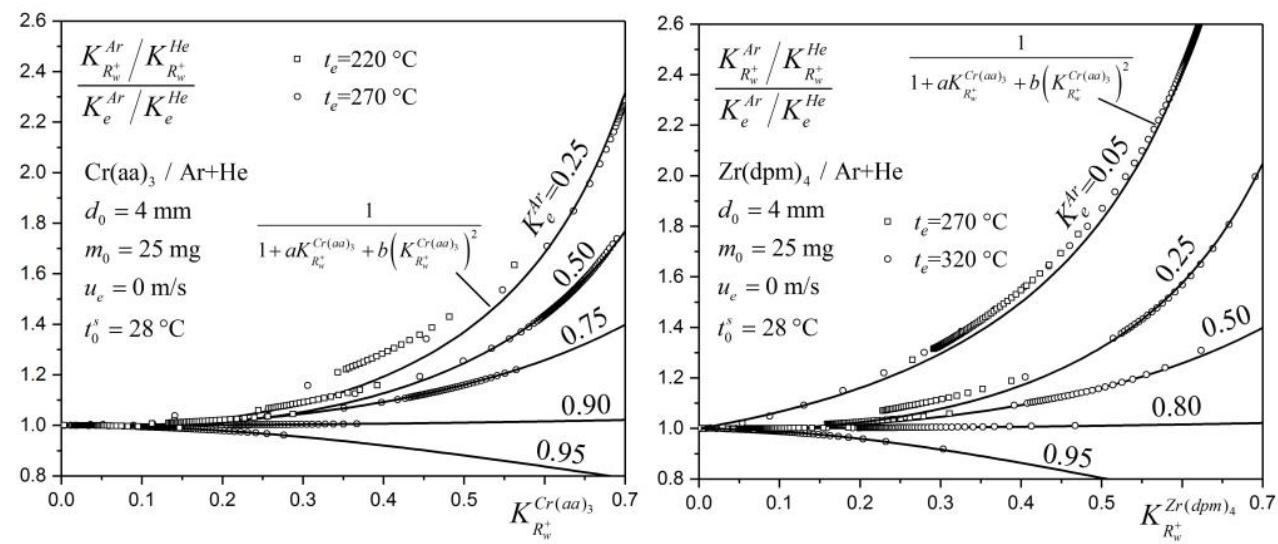

Fig. 1. The ratio of inert components $\mathrm{Ar}$ and $\mathrm{He}$ at sublimation of the particles of $\mathrm{Cr}(\mathrm{aa})_{3}$ and $\mathrm{Zr}(\mathrm{dpm})_{4}$ : points - simulation data, and lines - approximation for subsequent calculations in the forced convection mode.

Fig. 2 presents the results of calculations of mass flow rates of precursor vapors depending on time. It is seen that a small weight addition of helium to argon leads to a significant increase in the intensity of the mass transfer and a decrease of the full time of 
sublimation. For example, at $K_{e}^{H e}=0.05$ the full time of sublimation is twofold less than at sublimation in pure argon for both precursors.
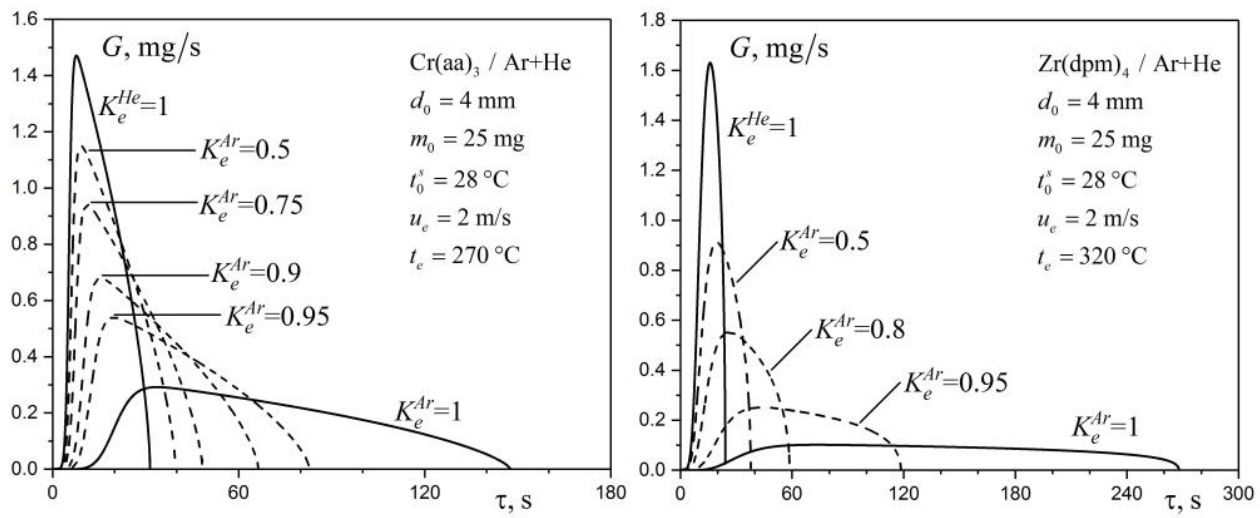

Fig. 2. The mass flow rate of $\mathrm{Cr}(\mathrm{aa})_{3}$ and $\mathrm{Zr}(\mathrm{dpm})_{4}$ vapors at the time.

\section{Conclusion}

Physical and mathematical model of nonstationary sublimation of a single particle of the MO CVD precursor floating in the gas-carrier flow with average pressure is presented. Numerical simulation of heat and mass transfer with $\operatorname{Cr}(\mathrm{aa})_{3}$ and $\operatorname{Zr}(\mathrm{dpm})_{4}$ precursors sublimation in the mixture of inert gases $\mathrm{Ar}$ and $\mathrm{He}$ has been carried out. The addition of helium to argon leads to a significant increase in the mass flow intensity and a decrease of the full time of sublimation.

The work was supported by the Russian Science Foundation (project No. 16-19-10325).

\section{References}

1. V.V. Krisyuk, A.E. Turgambaeva, I.K. Igumenov, Chem. vap. deposition 4, 43 (1998)

2. R.G. Parkhomenko, N.B. Morozova, G.I. Zharkova, Y.V. Shubin, S.V. Trubin, V.V. Kriventsov, B.M. Kuchumov, T.P. Koretskaya, I.K. Igumenov, Chem. vap. deposition 18, 336 (2012)

3. E.P. Volchkov, N.A. Dvornikov, V.V. Lukashov, R.K. Abdrakhmanov, Thermophysics and Aeromechanics 20, 663 (2013)

4. R.C. Reid, J.M. Prausnitz, T.K. Sherwood, The Properties of gases and liquids (McGraw-Hill, New York, 1977)

5. Yu.V. Lapin, M.Kh. Strelets, Internal flows of gas mixtures (Nauka, Moscow, 1989)

6. J.D. Ramshaw, Journal of non-equilibrium thermodynamics 15 (1990)

7. M.E. Ranz, W.R. Marshall, Chem Eng Prog 48, 141 (1952)

8. M.S. Makarov, S.N. Makarova, A.A. Shibaev, Proceedings of the Jubilee conference of National committee for heat and mass transfer of RAS and XXI School-seminar of young scientists and specialists under leadership of RAS academician A.I. Leontiev 2 (2017)

9. P.P. Semyannikov, I.K. Igumenov, S.V. Trubin, T.P. Chusova, Z.I. Semenova, Thermochim. Acta 432 (2005)

10. M. Fulem, K. Růžička, V. Růžička, T. Šimeček, E. Hulicius, J. Pangrác, Journal of Crystal Growth 264 (2004) 